\title{
Effects of Covid-19 Quarantine on Physical Activity and Psycho-Emotional Health
}

\author{
Ramunė Komžienė, ${ }^{1}$ Neda Valterytė, ${ }^{1}$ Milda Žukauskienė, ${ }^{1}$ \\ Laimutė Samsonienè, ${ }^{1,2}$ Laura Nedzinskiené ${ }^{2}$ \\ Vilniaus kolegija / University of Applied Sciences, ${ }^{1}$ Vilnius, Lithuania \\ Vilnius University, ${ }^{2}$ Vilnius, Lithuania
}

\begin{abstract}
Introduction. Quarantine is a complex definition that consists of administrative and sanitary measures designed to prevent the spread of communicable diseases to both humans and animals. Bans and restrictions that have been introduced, have significantly changed people's lifestyles - individuals have become less physically active and were forced to spend most of their time at home. The aim of the study was to reveal the dominant factors contributing to students' psycho-emotional health and physical activity during the quarantine period.

Methods. This survey was conducted in April 2020 with 304 participating students. The anonymous survey collected demographic data and two standardized questionnaires were used: The International Physical Activity Questionnaire and General Health Questionnaire. Data were processed by SPSS Statistics 23 program.

Results. Respondents' level of physical activity during quarantine was as follows: high level $-42 \%$, medium level $-41 \%$; low level $-17 \%$. A negative correlation was found - with increasing physical activity, the sum of psycho-emotional state assessment score decreased $(r=-.2702, p<.01)$, and with increasing age of respondents, their total level of physical activity also increased $(r=.1364, p<.05)$.

Conclusion. Low intensity of physical activity predominated at all levels of physical activity, for which the main reason was the remote learning during quarantine and recommendations to leave home only for essential matters. Regular physical activity and exercise may be a simple, inexpensive, and effective therapy during the quarantine, and physical activity is significant in treating student depression, stress, and/or increased anxiety.
\end{abstract}

Keywords: depression, youth, stress, sedentary lifestyle.

\section{INTRODUCTION}

$\mathrm{T}$ The global pandemic and quarantine restrictions applied in most countries have affected everyone, including students. Bans and restrictions were introduced changing the nature of their learning and work - forcing everyone to withdraw from active life and spend more time at home. The adverse circumstances have affected students' health during their studies, with some body systems (e.g., nerves) and organs being overloaded and others (cardiovascular and respiratory) suffering from a lack of physical activity (hypo-dynamics) (Booth, Roberts, \& Laye, 2012; Carlson, Fulton, Pratt, Yang, \& Adams, 2015). According to researchers (Jadhav, 2017), adequate physical activity is known to positively balance the psycho-emotional state at any human age. In the report, the World Health Organization (WHO, 2019) published a statement that exercise is an excellent preventative measure and an effective factor in the treatment of mental illness. Physical activity leads to an increased production of neurotransmitters like dopamine and serotonin, which are known to be lowered in several mental health conditions, as well as a higher number of newly generated neurons, which are important for learning and memory formation. According to US national surveys (Lipson, Lattie, \& Eisenberg, 2018), student mental health problems have increased by about $10 \%$ in the last 10 years. In 2018, according to research by the Lithuanian Students Union (Lithuanian 
Students Union, 2018a; Lithuanian Students Union, 2018 b), as many as $70 \%$ of students experienced stress and $83 \%$ were experiencing severe anxiety. As the research data (Macpherson, Teo, Schneider, \& Smith. 2017) suggest, regular physical activity helps control or reduce anxiety and stress, as well as contributes to greater self-confidence. The latest research (Baltic Survey, 2020) data revealed that $93 \%$ of Lithuanians were experiencing anxiety associated with the pandemic. According to the data of 2020 of the Lithuanian Center for Social Sciences, the pandemic situation affected the following areas of life the most: studies - by $95 \%$, leisure $-85 \%$, family life $-65 \%$, work $-64 \%$, and finance 55\%. According to 2017 Eurobarometer data (European Commission, 2018), 63\% of European Union students had a certain exercise routine, $10 \%$ exercised regularly, $11 \%$ infrequently, and $16 \%$ of students did not exercise at all. Insufficient physical activity and the introduction of new technologies offered new opportunities to promote physical activity and improve people's health, but these effects were not always positive and researchers as well as healthcare professionals were encouraged to further consider and analyse this contribution (Gao $\&$ Lee, 2019). The links between physical activity and psycho-emotional state have also been assessed in previous research, but there is a lack of data on the effects of quarantine during the pandemic on personal physical activity and psycho-emotional state. The aim of the study was to determine the dominant factors of students' physical activity and psycho-emotional health during quarantine.

\section{METHODS}

The survey was conducted in April 2020. The study participants were 306 students, the age of whom ranged from 18 to 42 years, with the average age being $24 \pm 17.8$ years $($ Median $=20$ ). The majority of the respondents were women $-88 \%$, and $12 \%$ men (every tenth of the respondents). Respondents were distributed according to study programs: general practice and nursing - 43\%, physiotherapy - $14 \%$, occupational therapy $10 \%$, radiology $-10 \%$, hygienic and decorative cosmetology, biomedical diagnostics, and dietetics with the remaining $23 \%$.

The anonymous survey collected demographic data and two standardized questionnaires were used:

- The International Physical Activity Questionnaire (IPAQ short version) - to measure the level and duration of physical activity of persons aged 18-69 during the week.

- General Health Questionnaire (GHQ-12) this questionnaire designed to assess the mental state of individuals and their recent emotions.

The study was conducted in accordance with all the principles of professional ethics, without violating the rights of the respondents and respecting the anonymity and confidentiality of the data obtained.

Data were processed by SPSS Statistics 23 program. Descriptive statistical methods were used for data analysis. To determine statistical significance, tests were applied to independent samples: for two samples - Student $t$-criterion and chi-square $(\chi 2)$. The results were evaluated as statistically significant when $p<.05$. The Pearson correlation coefficient was calculated to determine the strength of the statistical relationship.

Ethics approval and consent to participate in the study. The present study was approved by the Institutional Board of the Faculty of Health Care of the University of Applied Sciences. The aims of the survey were explained to students, who were free to participate or not participate, as denoted by the non-negligible nonresponse rate.

\section{RESULTS}

Assessing the physical activity of the respondents during the quarantine caused by the COVID-19 pandemic, it was revealed that physical activity (FA) was high (> 3000 MET- min/week) for $42 \%$ of all subjects in the study and for $41 \%$ - medium level (600-3000 MET- min/week). Other respondents $(17 \%)$ were in the group of low level of physical activity $(<600 \quad$ MET-min/week). No significant differences were observed in the distribution of FA groups by specialities. Assessing the intensity of physical activity of the respondents assigned to the group of low level of physical activity, it was observed that they mostly engaged in low intensity activity - walking (average $1 \mathrm{~d} /$ week, $19 \mathrm{~min} /$ day, $62.3 \%$ according to MET). Other intensity activities (respondents were significantly less engaged - neither high ( $0.1 \mathrm{~d} /$ week) nor medium $(0.3 \mathrm{~d} /$ week) intensity activities were performed full-time. Although both activities were distributed similarly, the distribution of medium-intensity activities according to MET was twice as high (27\%) compared to high intensity activities $(10.8 \%)$ 
Figure 1. Distribution of respondents according to the average physical activity intensity indicators in the low FA level group

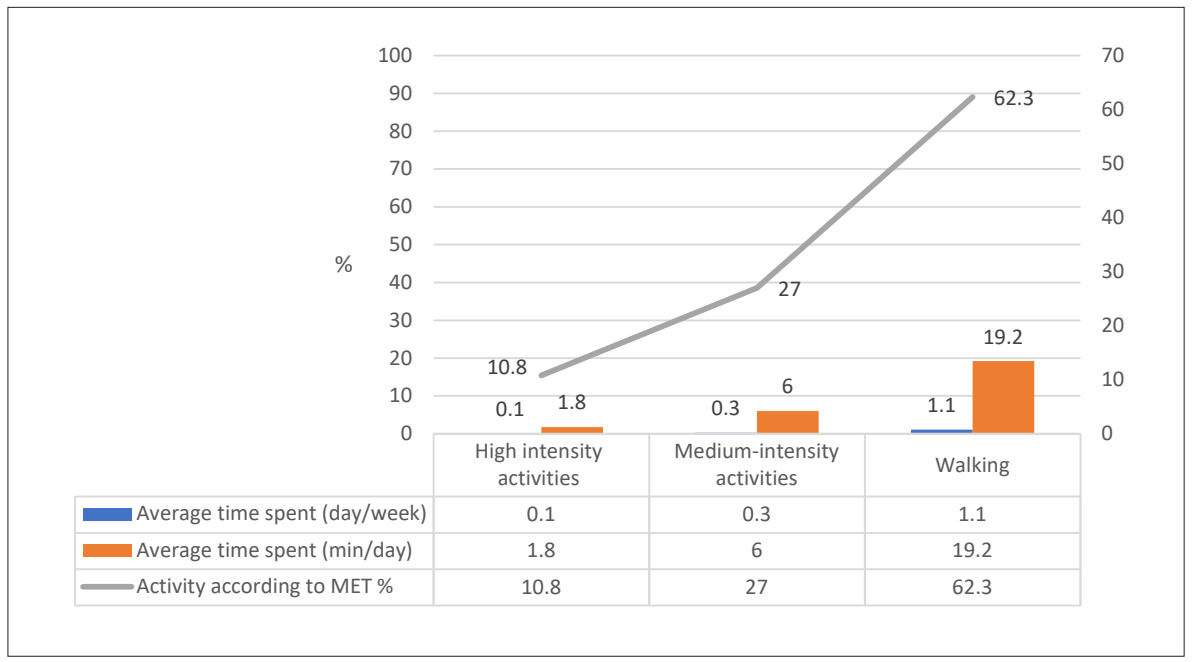

We also assessed the intensity of physical activity of the respondents assigned to the group of medium level of physical activity. The smallest part was occupied by high-intensity activities - the respondents spent on average 1 day/week, 24 minutes/ day engaging in this type of activity (MET $-17.2 \%$ ). Students engaged in medium-intensity activities twice as much as in high-intensity activities
Figure 2. Distribution of respondents according to the average physical activity intensity indicators in the group of medium FA level

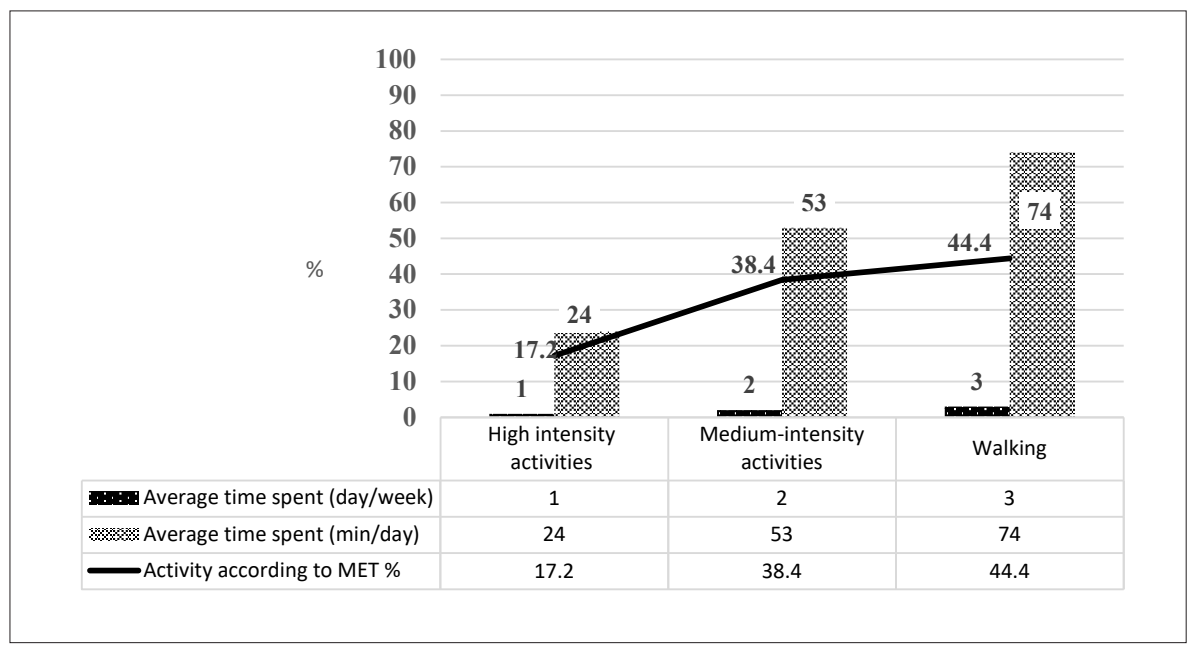

Respondents in the group of high level of physical activity were engaged in high intensity activities 2 days/week, 90 minutes/day on average (MET - 27\%). Respondents who engaged in medium-intensity activities performed them twice as many days (4 days/week) as high-intensity activities. It was discovered that walking accounted for the largest share of respondents' physical activity (5 days/week, 113 minutes/day, distribution according to MET $-32.3 \%$ )
Figure 3. Distribution of respondents according to the average physical activity intensity indicators in the group of high FA level

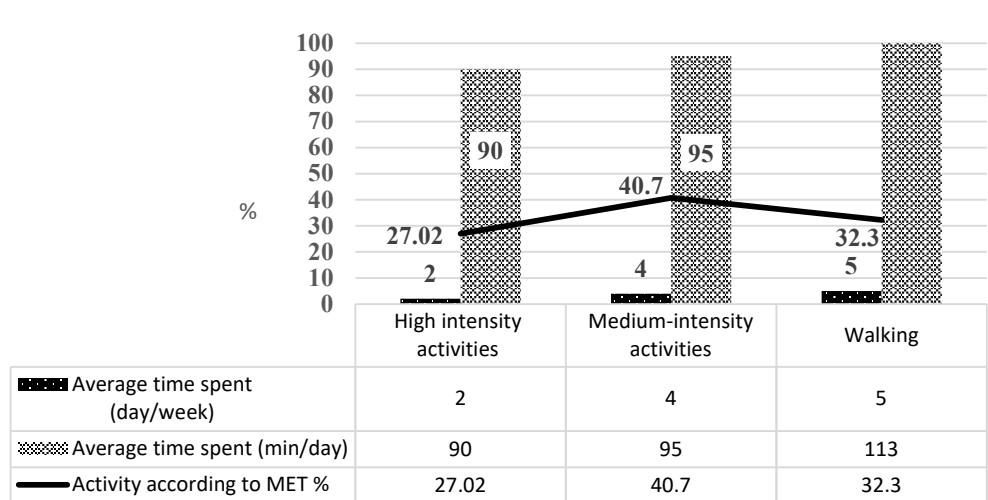


The research revealed that during the quarantine period, $55.6 \%$ of the respondents were exercising at home (following their individual routine). One third of the respondents (36.3\%) used technology, i.e., online workouts, YouTube platform; $17 \%$ of the respondents indicated that they did not exercise during the quarantine but had intentions to start and only $7.8 \%$ did not exercise and did not even have any intention to start.

Figure 4. Nature of respondents' physical activity during the quarantine period

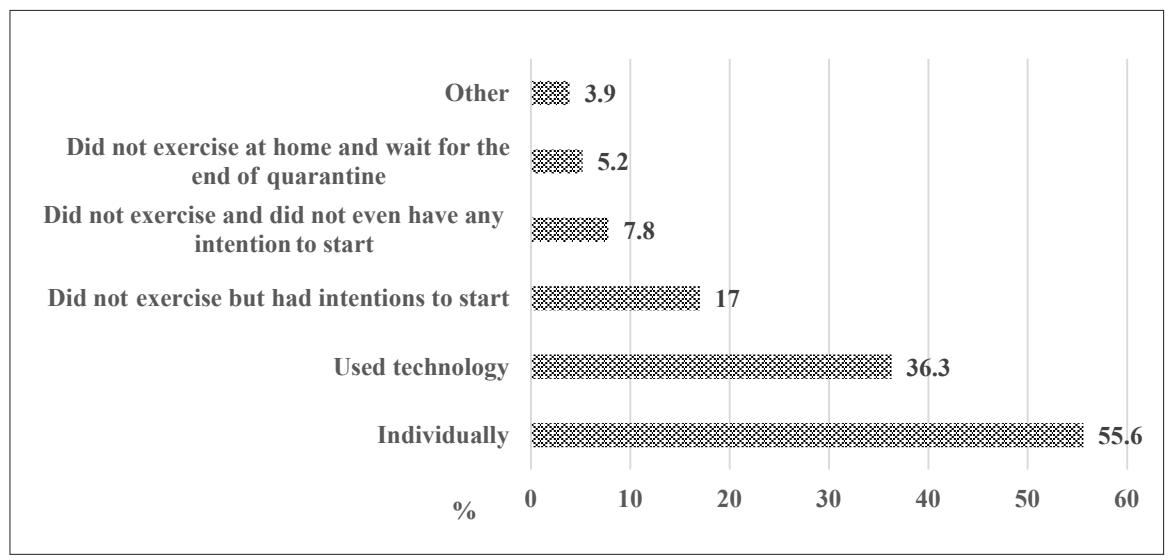

The evaluation of the measures used by the respondents to help overcome stress and/or anxiety revealed several activities. More than two thirds of respondents $(70.1 \%)$ declared that it was listening to music, $67.8 \%$ stated that watching movies and series was helpful, $64.5 \%$ respondents indicated that conversations with close people help them cope with stress.
Figure 5. Respondents' identified measures to reduce stress and/or anxiety

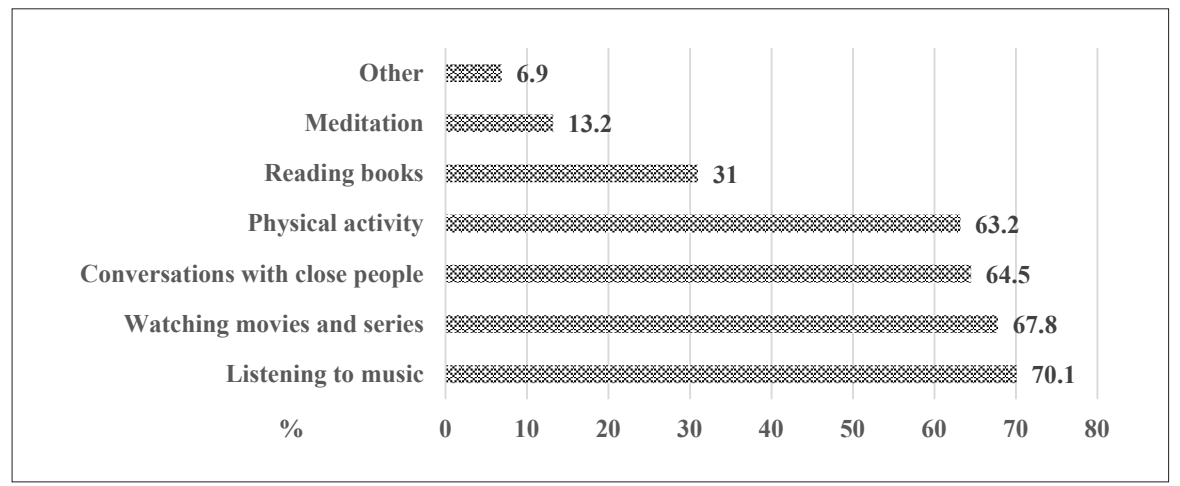

Assessing the correlation between physical activity and psycho-emotional state, a negative correlation was observed - with increasing physical activity, the sum of psycho-emotional state assessment scores decreased $(r=-.2702$, $p<.01$ ), and with increasing age of respondents, their total physical activity level increased $(r=.1364, p<.05)$. A negative correlation was also discovered between the passive time spent by the respondents and physical activity - the higher the physical activity, the shorter the sedentary time $(r=-.2844, p<.01)$.
Table 1. Correlation between respondents' age, physical activity, psychoemotional state and the amount of time spent sitting

Note. $* p<.05 ; \quad * * p<.01$

\begin{tabular}{|c|c|c|c|c|c|}
\hline \multicolumn{2}{|l|}{ Variables } & Age & $\begin{array}{l}\text { Physical } \\
\text { activity }\end{array}$ & $\begin{array}{c}\text { Passive time } \\
\text { spent (hour/day) }\end{array}$ & $\begin{array}{c}\text { Psycho-emotional } \\
\text { state }\end{array}$ \\
\hline Age & $\begin{array}{l}r \\
p\end{array}$ & 1 & & & \\
\hline Physical activity & $\begin{array}{l}r \\
p\end{array}$ & $\begin{array}{l}.1364 * \\
.0169\end{array}$ & 1 & & \\
\hline $\begin{array}{l}\text { Passive time spent } \\
\text { (hour/day) }\end{array}$ & $\begin{array}{l}r \\
p\end{array}$ & $\begin{array}{r}-.0858 \\
.1375\end{array}$ & $\begin{array}{l}-.2844 * * \\
.0001\end{array}$ & 1 & \\
\hline Psycho-emotional state & $\begin{array}{l}r \\
p\end{array}$ & $\begin{array}{l}-.1987^{* *} \\
.0004\end{array}$ & $\begin{array}{l}-.2702 * * \\
.0002\end{array}$ & $\begin{array}{l}.0882 \\
.1265\end{array}$ & 1 \\
\hline
\end{tabular}


A statistically significant negative correlation ( $p=.0004)$ was found between low-intensity physical activity and psycho-emotional status $(r=-.2025)$. A positive but very low correlation $(r=.0243)$ between the average level of physical activity and psycho-emotional status was also found; however, this correlation was not statistically significant. The research data revealed that with the increase of general level of physical activity of the respondents, the feeling of constant tension decreased $(r=-.1921, p<.01)$.

Table 2. Correlations between respondents' total physical activity, depressed mood and constant tension

Note. $* p<.05 ; \quad * * p<.01$

\begin{tabular}{|c|c|c|c|c|c|}
\hline \multicolumn{2}{|c|}{ Variables } & Age & $\begin{array}{l}\text { Physical } \\
\text { activity }\end{array}$ & $\begin{array}{c}\text { Depressed } \\
\text { mood }\end{array}$ & Stress \\
\hline Age & $\begin{array}{l}r \\
p\end{array}$ & 1 & & & \\
\hline Physical activity & $\begin{array}{l}r \\
p\end{array}$ & $\begin{array}{l}.1295^{*} \\
.0235\end{array}$ & 1 & & \\
\hline Depressed mood & $\begin{array}{l}r \\
p\end{array}$ & $\begin{array}{c}-.2716^{* *} \\
.0001\end{array}$ & $\begin{array}{c}-.2480 * * \\
.0011\end{array}$ & 1 & \\
\hline Stress & $\begin{array}{l}r \\
p\end{array}$ & $\begin{array}{r}-.0491 \\
.3918\end{array}$ & $\begin{array}{c}-.1921 * * \\
.0726\end{array}$ & $\begin{array}{l}.5438^{* *} \\
.0011\end{array}$ & 1 \\
\hline
\end{tabular}

It was observed that higher levels of physical activity had an inverse association with depressed $\operatorname{mood}(r=-.2480, p<.01)$. A negative correlation was also found between the respondents' elderly age and depressed mood $(r=-.2716, p<.01)$. The relationship between respondents' age and stress was not statistically significant.

\section{DISCUSSION}

Undergraduate students have shown a tendency to be less physically active due to new stresses in life, including higher academic demands and financial concerns (Joseph, Royse, Benitez, \& Pekmezi, 2014). Stressors are divided into three types according to the nature of the events: disasters, important life changes, and daily difficulties. During the quarantine, additional restrictions on movement and restrictions on mutual communication turned the students' daily agendas less dynamic. Additional everyday difficulties appeared, such as remote learning or recommendations to go outside only when necessary, limited physical or communicational contact or lack of contact with close people, also other applied restrictions.

An optimal physical activity is one of the health-enhancing factors, and long-term movement restrictions as well as physical immobility during the quarantine are of great concern for the physical, mental and social health of young people. The emergence of new mild stressors accumulates over time and may lead to health changes. Researchers (Booth et al., 2012; Carlson et al., 2015; WHO, 2010) identify sedentary as a cause of many chronic noncommunicable diseases, as well as a determinant of population mortality.
WHO (2019) encourages all individuals to follow the physical activity guidelines provided, which can be performed at home without applying any additional measures. According to the study, majority of respondents did not follow the WHO physical activity recommendations during the quarantine at home. In order to conform to the quarantine recommendations, the majority of our surveyed respondents engaged in low-intensity physical activity - walking, and $17 \%$ of the respondents were classified as practicing low physical activity, $14 \%$ of the subjects did not exercise at all. The data obtained on student absenteeism are corresponding to the Eurobarometer data of 2017 (European Commission, 2018). It is important to note that chronic fatigue syndrome develops due to insufficient or extensive exercise, stress, lack of sleep, various illnesses or injuries. An important factor, contributing to reduced fatigue and increased ability to work for the students, would be the adjustment of physical activity. Higher levels of physical activity have shown to have greater positive effects on mental health than moderate to low levels of physical activity (Bhochhibhova, Branscum, Taylor, \& Hofford, 2014), as well as more frequent and higher levels of physical activity are more efficient to mental health and cognitive function in young adults than low levels of physical activity (walking) (Nakagawa et al., 2020).

According to the data obtained from the GHQ-12 questionnaire, $83 \%$ of the respondents scored more than 12 points, which is the threshold above which they might be considered to have had certain psychological problems during quarantine. Assessing the respondents' subjective opinions about their mood, emotions and general 
well-being, it was observed that most respondents had depressed mood, constant tension, loss of selfconfidence, had difficulty in coping with various problems, also complained of sleep disorders, stress, low self-esteem and difficulties to concentrate. It may be concluded that stress experienced by the respondents, the psychological tension and the abundance of work during quarantine is a certain kind of imbalance between the students' health and unforeseen daily stresses in life caused by unexpected changes in their agenda. If stressful conditions are frequent or persist for a long time, the body's reserves are depleted and in the long term it may lead to various diseases: hypertension, heart attack, diabetes, depression, and oncological diseases. The negative association between low physical activity and psycho-emotional state revealed in our study coincides with the findings of other researchers (Basso \& Suzuki, 2017) that physical activity influences better mood and decreases stress levels. We obtained data similar to those of other researchers in non-quarantine conditions - a statistically significant positive correlation between respondents' age and physical activity and a negative correlation between physical activity and psycho-emotional health. A metaanalysis of the literature by Schuch et al. (2018) suggests that increased physical activity provides a protective effect on the future development of depression in people of all ages.

According to the study data, one third of the respondents did not express categorical opinions that their mental well-being changed significantly, they rather stated that they could enjoy everyday life, pleasant routines (reading a book or cooking) more than usual. Such changes may have been caused by the increased amount of free time during the quarantine and the high level of physical activity. The results of our study correspond to the data obtained by other researchers (Chow \& Choi, 2019; Kavosi et al., 2015) that physical activity not only reduces symptoms of stress, anxiety, or depression, but also increases self-esteem and self-value, which helps maintaining good psycho-emotional status.
Summarizing the results of the study during the quarantine period and comparing it with the data of other researchers obtained during non-quarantine, it may be stated that there are links between physical activity, psycho-emotional status and age. Physical activity helps to effectively reduce the symptoms of stress, nervous tension. Data from many studies suggest that physical activity is strongly correlated with anxiety and its reduction, and physical activity helps to increase self-esteem and self-value. Under certain circumstances (e.g., during the quarantine), personal perception about controlling the situation is detrimental to their health. We therefore would like to draw attention to the lack of physical activity of students during the COVID-19 pandemic considering all the possible consequences. It is important to note that in order to change students' behaviour during quarantine, it is important to assess their motivation to exercise or not to practice sports, and only then offer and/ or adapt physical activity measures that are least stressful and most attractive.

\section{CONCLUSIONS}

All respondents spent the majority of their day sitting and/or walking. The main reason for the reduced movement activity is remote learning during the quarantine and recommendations to leave home only when needed. Insufficient intensity of respondents' physical activity and poor agenda in the long term may be the cause of various diseases. To cope with stress and anxiety, most of the respondents chose different means during the quarantine - artistic, intellectual and/or physical activities. Therefore, regular physical activity and exercise may be a simple, inexpensive, and effective therapy during the quarantine, and physical activity is significant in treating student depression, stress, and/or increased anxiety.

Funding.This study did not receive any specific grant from funding agencies in the public, commercial, or not-for-profit sectors.

Conflict of interest. The authors declare that they have no conflict of interest.

\section{REFERENCES}

Baltic Survey. (2020). Moods of the Lithuanian population: anxiety about family members and their health, long fight against the pandemic. Retrieved from: https://www.baltic-surveys.lt/covid-19-ekspresasnuotaikos
Basso, J. C., \& Suzuki, W. A. (2017). The effects of acute exercise on mood, cognition, neurophysiology, and neurochemical pathways: a review. Brain Plasticity, 2(2), 127-152. https://doi.org/10.3233/BPL-160040 
Bhochhibhova, A., Branscum, P., Taylor, E.L. \& Hofford, C. (2014). Exploring the relationships of physical activity, emotional intelligence, and mental health among college students. American Journal of Health Studies, 29, 191-197.

Booth, F. W., Roberts, C. K., \& Laye, M. J. (2011). Lack of exercise is a major cause of chronic diseases. Comprehensive Physiology, 2(2), 1143-1211. https:// doi.org/10.1002/cphy.c110025

Carlson, S. A., Fulton, J. E., Pratt, M., Yang, Z., \& Adams, E. K. (2015). Inadequate physical activity and health care expenditures in the United States. Progress in Cardiovascular Diseases, 57(4), 315-323. https://doi. org/ 10.1016/j.pcad.2014.08.002

Chow, S. K. Y., \& Choi, E. K. Y. (2019). Assessing the mental health, physical activity levels, and resilience of today's junior college students in self-financing institutions. International Journal of Environmental Research and Public Health, 16(17), 3210. https://doi. org/10.3390/ijerph16173210

European Commission (2018). Public Opinion. Special Eurobarometer 472: Sport and physical activity in 2017. Retrieved from: https://ec.europa.eu/sport/sites/default/ files/special-eurobarometer-472_en.pdf

Gao, Z., \& Lee, J. E. (2019). Emerging technology in promoting physical activity and health: Challenges and opportunities. Journal of Clinical Medicine, 8, 1830. https://doi.org/10.3390/jcm8111830

Jadhav, R. R. (2017). The effect of exercise on anxiety of college students. International Journal of Physical Education, Sports and Health, 4, 231-233.

Joseph, R. P., Royse, K. E., Benitez, T. J., \& Pekmezi, D. W. (2014). Physical activity and quality of life among university students: exploring self-efficacy, self-esteem, and affect as potential mediators. Quality of Life Research, 23(2), 659-667. https://doi.org/10.1007/ s11136-013-0492-8

Kavosi, A., Saadati, M., Movahedi, A., Farahnia, M., Mohammadi, G., Aghababayan, A., ... \& Assari, S. (2015).
Physical activity enhances self-esteem of male college students; a randomized controlled trial. International Journal of Travel Medicine and Global Health, 3(2), 49-52. https://dx.doi.org/10.20286/ijtmgh-030298

Lipson, S. K., Lattie, E. G., \& Eisenberg, D. (2019). Increased rates of mental health service utilization by US college students: 10-year population-level trends (2007-2017). Psychiatric Services, 70(1), 60-63. https:// doi.org/10.1176/appi.ps.201800332

Lithuanian Center for Social Sciences. (2020). Threats and new opportunities. Retrieved from http://www.dsti. lt/index_en.php

Lithuanian Students Union (2018a). Activity report 2017-2018. Vilnius.

Lithuanian Students Union (2018b). Satisfaction with studies and psychological health of students. Retrieved from https://lsp.lt/en/apie-lss

Macpherson, H., Teo, W. P., Schneider, L. A., \& Smith, A. E. (2017). A life-long approach to physical activity for brain health. Frontiers in Aging Neuroscience, 9, 147. https://doi.org/10.3389/fnagi.2017.00147

Nakagawa, T., Koan, I., Chen, C., Matsubara, T., Hagiwara, K., Lei, H., ... \& Nakagawa, S. (2020). Regular moderate-to vigorous-intensity physical activity rather than walking is associated with enhanced cognitive functions and mental health in young adults. International Journal of Environmental Research and Public Health, 17(2), 614. https://doi.org/10.3390/ijerph17020614

Schuch, F. B., Vancampfort, D., Firth, J., Rosenbaum, S., Ward, P. B., Silva, E. S., ... \& Stubbs, B. (2018). Physical activity and incident depression: a meta-analysis of prospective cohort studies. American Journal of Psychiatry, 175(7), 631-648. https://doi.org/10.1176/ appi.ajp.2018.17111194

WHO (2010). Global Recommendations of Physical Activity for Health. World Health Organization.

WHO (2019). Motion for your mind. Physical activity for mental health promotion, protection and care. World Health Organization. 